\title{
MICROBOLOMETERS WITH ADAPTIVE DETECTIVITY USING ELECTROSTATIC ACTUATION*
}

\author{
Woo-Bin Song and Joseph J. Talghader \\ Department of Electrical and Computer Engineering, University of Minnesota \\ Minneapolis, MN 55455
}

\begin{abstract}
The first microbolometers with electronically controlled detectivities are reported. Such devices are needed to thermally image scenes containing both very high and low temperatures. In the high detectivity state, the microbolometers are operated similar to standard commercial devices [1]. In the low detectivity state, portions of the support beams are brought in contact with the substrate to reduce the sensitivity of the devices. This can be done on a pixel-by-pixel basis. In low voltage testing, the maximum actuation voltage is set to $17 \mathrm{~V}$, and the detectivities of a typical device can be switched more than an order of magnitude between $1.8 \times 10^{6} \mathrm{cmHz}^{1 / 2} / \mathrm{W}$ and $1.5 \times 10^{5} \mathrm{cmHz}^{1 / 2} / \mathrm{W}$. The device pixel size is $100 \mu \mathrm{m}$ by $100 \mu \mathrm{m}$ and is designed for an array fill factor of $91 \%$.
\end{abstract}

\section{INTRODUCTION}

Thermal detectors based on small bandgap materials such as $\mathrm{Hg}_{0.8} \mathrm{Cd}_{0.2} \mathrm{Te}$ (MCT), InSb, and others have been studied and commercialized primarily for military and space science applications [2]. These photon detectors have very high performance but require cryogenic cooling systems to reduce parasitic dark currents.

With the advent of micromachining technology, it was realized that sufficiently high performance could be achieved with uncooled detectors that operated based on the heating of a small mass. Several classes of devices for reading the rise in temperature were developed. First and most prominent of these was the bolometer[3,6], but also developed were pyroelectric detectors [4], thermoelectric detectors [5], and others. The cost and weight of these devices is dramatically reduced compared to infrared photon detectors because cooling is no longer needed and because the materials involved are much easier to work with. For example, Wood, et. al. [6] reported high-quality infrared imaging in an infrared focal plane array using a monolithic silicon-based process. Recent studies have attempted to improve the performance of the basic microbolometer by developing high temperature-coefficientof-resistance (TCR) materials such as semiconducting $\mathrm{YBaCuO}$ (which is well-known for its superconducting superconducting state in other technologies) [7], vanadium oxide [8], and so on. Also new device structures have been proposed to enhance sensitivity and thermal isolation [9].

Typical thermal detectors are designed for high thermal isolation, and this makes them extremely receptive to small differences in background temperature. However, high sensitivity potentially has drawbacks when a scene contains very bright areas that may results from fires, explosions, or engine operation. For example, the most common material used as the TCR layer in microbolometers, vanadium oxide, is thermochromic, meaning that its absorption and resistance properties change if the microbolometer is heated excessively. Also, the electronics used to amplify the signals at the edges of the array would be unable to handle the full dynamic range needed to image the smallest and brightest signals. Intensity-dependent noise and device damage are other possible issues. In this paper, an adaptive microbolometer is shown that is able to handle both high and low intensity signals. It does not require major changes to standard commercial architectures but rather uses an already-existing electrostatic actuator between the ground electrode and detector plate to bring a small area of the supports into contact with the substrate, thereby increasing device thermal conductance. To characterize this adaptive microbolometer, measurements of thermal conductance, responsivity, and detectivity have been made with and without electrostatic actuation.

\section{BACKGROUND}

Since the responsivity of a thermal detector is inversely proportional to its thermal conductance, the detectors must be thermally isolated from their surroundings. As implied in the previous section, a good thermal isolation can be achieved by having an air gap between the sensing element and the substrate with only thin support beams connecting them. To insure that the thermal conductance is determined by the support design, detector arrays are packaged under low vacuum conditions in hermetically sealed packages.

Previous research in our group has shown that the thermal conductance $[\mathbf{1 0 , 1 1}]$ and thermal responsivity $[\mathbf{1 1}]$ of micromachined beams and plates can be tuned both continuously and discretely. Continuous tuning of the responsivity was demonstrated over a factor of three and discrete tuning over almost two orders of magnitude. It was shown that the fundamental limit of thermal conductance tuning was about 4 orders of magnitude limited by the thermal contact conductance on the high end and radiation heat transfer on the low end.

While these initial studies were suggestive, the devices used in the demonstration were not designed for high fill factor or high thermal isolation. In addition, it is ultimately the detectivity of a detector that determines performance. The detectivity can be calculated from the expression,

$$
D^{*}=\frac{(A)^{1 / 2}\left(\Delta f_{\text {bandwidth }}\right)^{1 / 2} \mathfrak{R}_{V}}{{\sqrt{\bar{V}_{\text {noise }}}}^{2}}
$$

where $R_{\mathrm{V}}$ is the responsivity, A is the area of a pixel plate, $\Delta f_{\text {bandwidth }}$ is the bandwidth of measurement, and $\overline{V_{\text {noise }}^{2}}$ is the mean square value of the fluctuating noise voltage.

\footnotetext{
* Travel support has been generously provided by the Transducers Research Foundation and by the DARPA MEMS and DARPA BioFlips programs.
} 


\section{EXPERIMENT AND DISCUSSION}

The devices constructed for this study were microbolometers that used an inherent electrostatic actuator to bring its support beams into partial contact with the substrate as shown in Fig. 1. This induced a large change in the thermal conductance of the devices which directly led to similar changes in responsivity and detectivity. The thermal contact conductances of the materials interfaces used in micromachining are high enough [12] so that touching a small portion is sufficient to can cause dramatic changes in detectivity. While total thermal contact could change the potential dynamic range by four orders of magnitude [13], most imaging requires only a fraction of this range. Partial contact also helps to eliminate stiction during operation. Relatively low actuation voltages can be used due to the low rigidity of the supports.

The adaptive microbolometers are composed of two layers of silicon nitride structural material separated from the substrate and each other by polyimide sacrificial layers. First a $500 \mathrm{~nm}$ silicon nitride layer is deposited on top of a highly doped silicon wafer for electrical insulation followed by $1500 \mathrm{~nm}$ of spin-coated polyimide, which is patterned and etched with an $\mathrm{O}_{2}$ plasma to form anchor points for the 500nm thick PECVD nitride support beams. The support beams are coated with $\mathrm{Ti} / \mathrm{Au}$ to insure a low resistance for read-out. Then, a second polyimide layer is spincoated, patterned, and etched with an $\mathrm{O}_{2}$ plasma to make vias from the Ti/Au to the TCR material (Ti) of the microbolometer plate, which is deposited later. The pixel plate is composed of a $100 \mathrm{~nm}$ Ti layer, which is the TCR material, sandwiched between two 200nm PECVD nitride layers. Between depositions, vias are opened from the $\mathrm{Ti}$ to the $\mathrm{Ti} / \mathrm{Au}$ of the supports. In the final step, the double polyimide layers are isotropically etched in an $\mathrm{O}_{2}$ plasma to release the pixel plate and its support beams from the substrate. The designed pixel is $100 \mu \mathrm{m}$ by $100 \mu \mathrm{m}$ and has an array fill factor of $91 \%$. Images of completed devices and a small test array are shown in Fig. 2. Typical device characteristics are given in Table I.

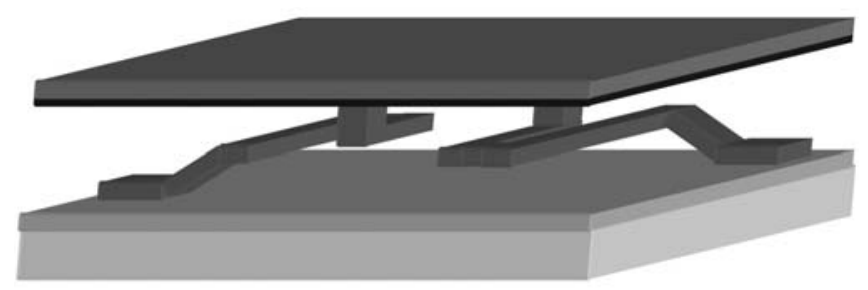

(a)

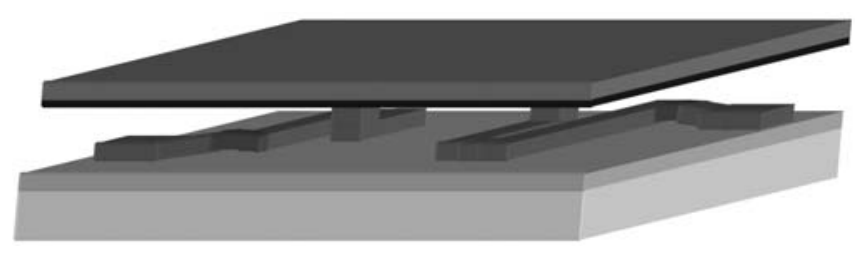

(b)

Figure 1. Conceptual diagram of a tunable detectivity microbolometer. In diagram (a), the device stands in a state of low thermal conductance and high detectivity. In diagram (b), the device supports have been actuated into contact with the substrate, creating a high thermal conductivity and low detectivity state. In practice only the edge farthest from the ground support contacts the substrate to prevent stiction.

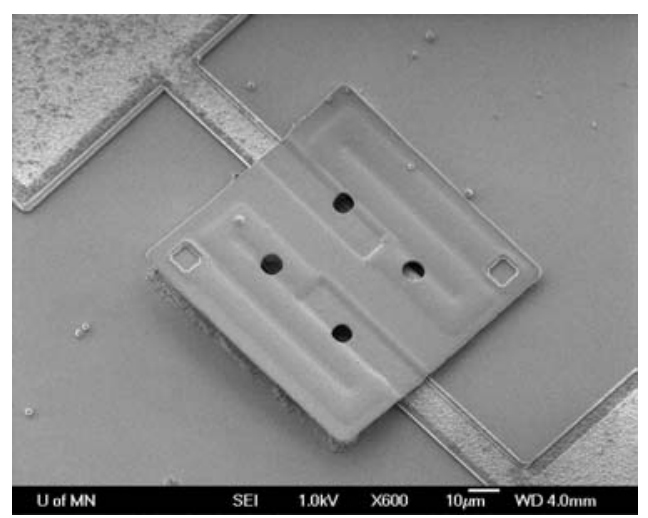

(a)

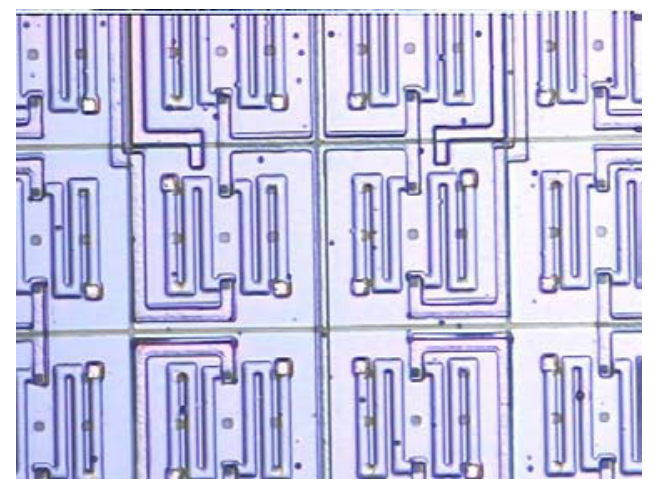

(b)

Figure 2. (a) SEM image of an individual microbolometer device. The microbolometer pixel dimensions are designed to be compatible with high fill-factor arrays and shown in part (b), an optical image of a portion of a $4 \times 4$ high fill factor array.

\begin{tabular}{c|c}
\hline Parameter & Value \\
\hline Size of Pixel plate & $100 \mu \mathrm{m}$ x $100 \mu \mathrm{m}$ \\
\hline \multirow{3}{*}{ Thickness of Pixel plate } & $\begin{array}{c}\text { Top plate (PECVD Nitride): } \\
0.2 \mu \mathrm{m}\end{array}$ \\
\cline { 2 - 2 } & $\begin{array}{c}\text { TCR layer (Titanium): } \\
0.1 \mu \mathrm{m}\end{array}$ \\
\hline Length of Support beam & $0.2 \mu \mathrm{m}$ \\
\hline Width of Support beam & $277 \mu \mathrm{m}$ \\
\hline $\begin{array}{c}\text { Thickness of } \\
\text { Support beam }\end{array}$ & $6 \mu \mathrm{m}$ \\
\hline Air Gap Spacing & $0.5 \mu \mathrm{m}$ \\
\hline $\begin{array}{c}\text { Thermal Coefficient of } \\
\text { Resistance }\end{array}$ & \begin{tabular}{c} 
Plate to support beam: $1.5 \mu \mathrm{m}$ \\
\hline
\end{tabular} \\
\hline
\end{tabular}




\begin{tabular}{c|c}
$\begin{array}{c}\text { Thermal Conductivity of } \\
\text { PECVD Nitride }\end{array}$ & $0.038 \sim 0.051 \mathrm{~W} / \mathrm{cm}-\mathrm{K}$ \\
\hline $\begin{array}{c}\text { Density of } \\
\text { PECVD Nitride }\end{array}$ & $2.22 \mathrm{~g} / \mathrm{cm}^{3}$ \\
\hline $\begin{array}{c}\text { Specific Heat of } \\
\text { PECVD Nitride }\end{array}$ & $1.31 \sim 1.73 \mathrm{~J} / \mathrm{g}-\mathrm{K}$ \\
\hline Heat Capacity of Device & $2.55 \times 10^{-8} \mathrm{~J} / \mathrm{K}$ \\
\hline Thermal Time Constant & $1.5 \mathrm{msec}$
\end{tabular}

Table I. Measured and calculated parameters for the microbolometer whose data is shown in Figs. 3, 4, and 5.

The thermal conductances of the devices were measured under vacuum $(<10$ mTorr) using a standard electrical method described in [14]. In this technique, a current is run through the detector plate which heats the device and changes the device resistance. The amount of heating caused by a given current depends on the thermal conductance of the device, which is extracted from a plot of $1 / \mathrm{R}$ vs. $\mathrm{I}^{2}$, as shown in Fig. 3. A typical device gives a thermal conductance, $\mathrm{G}=1.68 \times 10^{-5} \mathrm{~W} / \mathrm{K}$ when unactuated, and $\mathrm{G}=2.99$ $\mathrm{x} 10^{-4} \mathrm{~W} / \mathrm{K}$ using an actuation voltage of $17 \mathrm{~V}$.

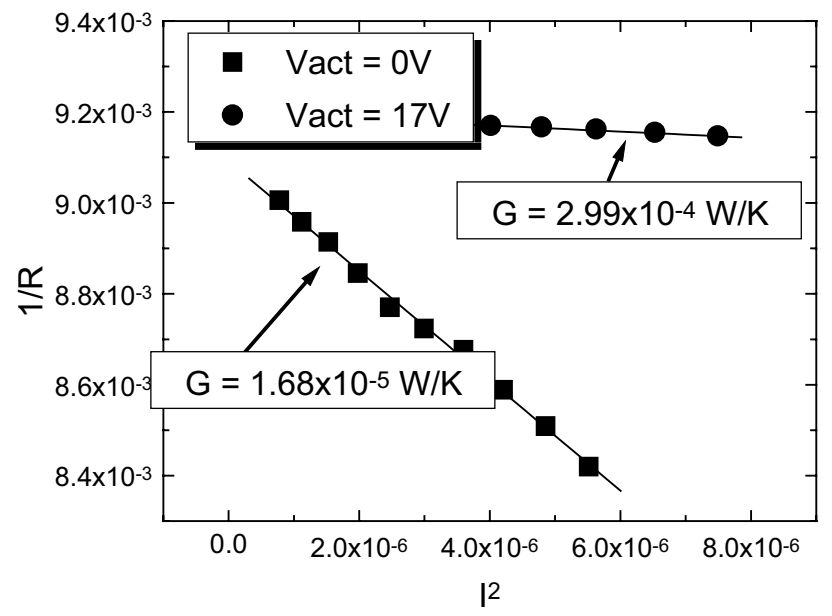

Figure 3. A plot of the inverse resistance versus current squared for a microbolometer in its unactuated $($ Vact $=0 \mathrm{~V})$ and soft actuation $($ Vact $=17 \mathrm{~V})$ states. This type of plot is commonly used for microbolometers because the slope is inversely proportional to the thermal conductance. The plot shows that the thermal conductance of a device is changed by more than an order of magnitude even in a soft actuation state.

Responsivity and detectivity measurements were also made. The devices were optically heated by a semiconductor laser of $\lambda=$ $658 \mathrm{~nm}$. The frequency of the signal was scanned by directly modulating the laser. The incident intensity was correlated with a detector and to the area of a pinhole aperture that was near to the size of the device under test. The microbolometer output voltage was amplified using a PARC113 preamplifier and examined with an HP35660A dynamic signal analyzer.

The responsivity of the same device from Fig. 3 is shown in Fig. 4. The responsivity varies by over an order of magnitude when the support beam of the device snaps down at a voltage of 17 $\mathrm{V}$. The reponsivities of the device taken at frequencies below $10 \mathrm{~Hz}$ show substantial scatter because of $1 / \mathrm{f}$ noise in our experimental set-up and are therefore not plotted.

Once the responsivity had been measured, the noise spectrum of the devices was obtained directly from the signal analyzer and used to extract the detectivity as described in Eq. 1. Fig. 5 shows the detectivity vs. frequency of the same device as in Figs. 3 and 4. The detectivity varies more than an order of magnitude between $1.8 \times 10^{6} \mathrm{cmHz}^{1 / 2} / \mathrm{W}$ and $1.5 \times 10^{5} \mathrm{cmHz}^{1 / 2} / \mathrm{W}$, in $0 \mathrm{~V}$ actuation and $17 \mathrm{~V}$ actuation, respectively. As mentioned above, the detectivities below $10 \mathrm{~Hz}$ were not plotted because of difficulties in measuring $1 / \mathrm{f}$ noise at extremely low sampling rates, which made the uncertainty in the detectivity values very large. This problem might be solved by replacing the Ti TCR material with $\mathrm{VO}_{\mathrm{X}}$, because $\mathrm{VO}_{\mathrm{X}}$ gives a relatively low $1 / \mathrm{f}$ noise characteristic at low frequency range as well as a high TCR of $-2.0 \% / \mathrm{K}[6]$. In this measurement, Johnson noise was around $120 \mathrm{nV} / \mathrm{Hz}^{1 / 2}$, which is somewhat higher than in typical microbolometers. This noise will be reduced in the future with a low-noise resistor for electrical read-out and a high TCR element.

The devices described are the first microbolometers made with a fully adaptable detectivity, but they have a relatively low performance relative to commercial devices. In particular, the device was designed to have a much lower thermal conductance of $7 \times 10^{-7} \mathrm{~W} / \mathrm{K}$ than measured in its unactuated state. This would directly translate to another 1.5 orders of magnitude improvement in responsivity and detectivity. It is suspected that the top layer of polyimide between the plate and the support beam was not fully removed during etch-release. This will be corrected in future processing. In addition, the performance could be further improved by using vanadium oxide instead of titanium as the TCR layer. Commerical VOx has a TCR that is approximately $-2 \% / \mathrm{K}$ compared to a TCR for Ti of approximately $0.2 \% / \mathrm{K}$ as shown in Fig. 6. It additionally has a very low $1 / \mathrm{f}$ noise, indicating further improvement. With these changes the architecture presented here could provide commercial thermal imaging performance with full intensity adaptivity, extending the dynamic range of present-day microbolometers by multiple orders of magnitude.

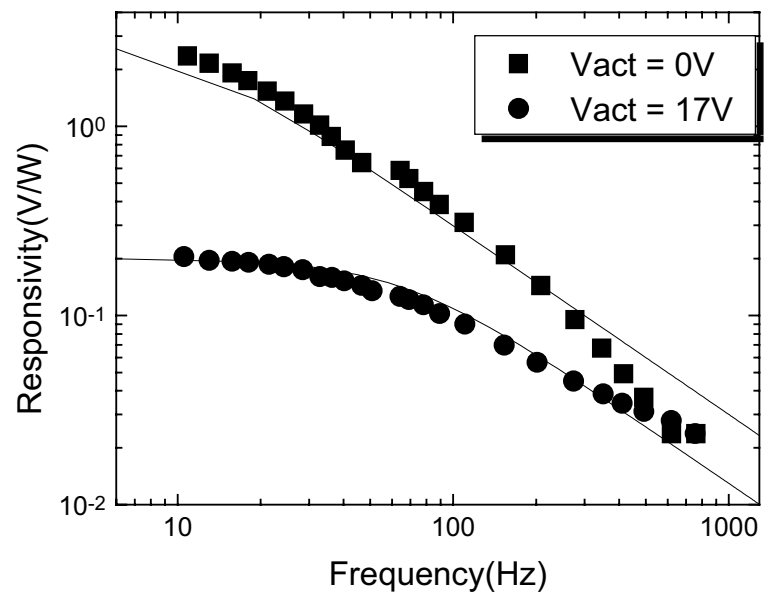

Figure 4. Responsivity vs. frequency for an adaptive detectivity microbolometer. The response varies by over an order of magnitude when the lowest device support is snapped down at $17 \mathrm{~V}$. Note that frequencies below $10 \mathrm{~Hz}$ were not plotted for consistency with Fig. 5 (see explanation there). The responsivity is artificially low because of the support beam widths and metal thicknesses used in the microbolometer are higher than would be used in a production environment. This represents limitations of 
our available fabrication processes rather than the architecture itself.

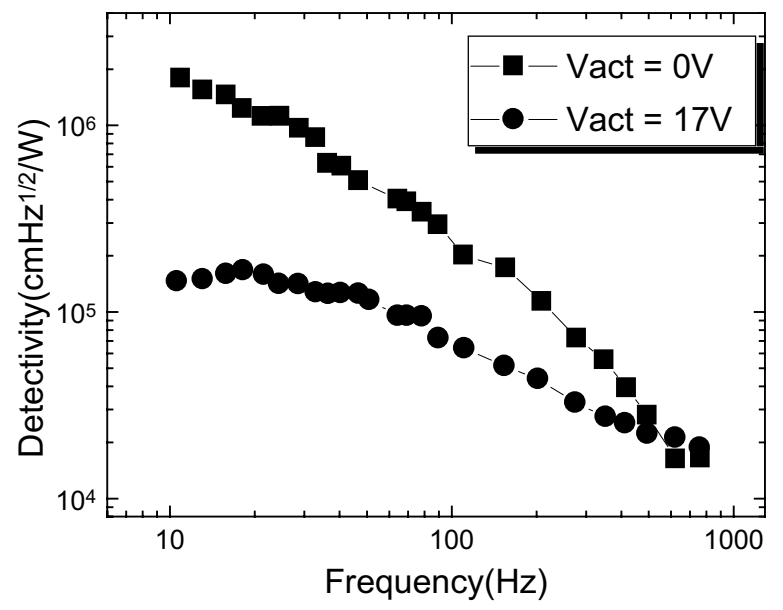

Figure 5. Detectivity vs. frequency for a typical device. The detectivity is in a high state at $0 \mathrm{~V}$ actuation and a low state at $17 \mathrm{~V}$ actuation. Frequencies below $10 \mathrm{~Hz}$ were not plotted because of the difficulties in measuring $1 / f$ noise at extremely low sampling rates, which made the uncertainty in the detectivity values large at very low frequencies.

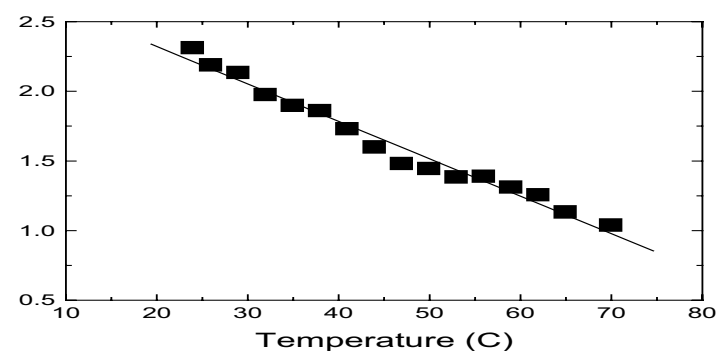

(a) VO, $\mathrm{O}_{x}$,unit of Resistance in $x$-axis: Mohm

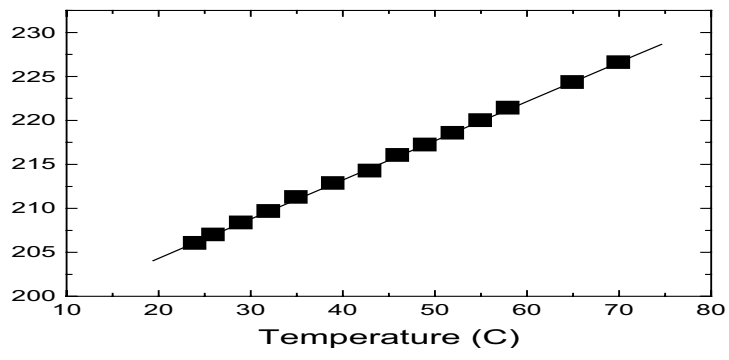

(b) Ti, unit of Resistance in $x$-axis: ohm

Figure 6. Resistance vs. temperature measured by pointprobe for VOx (a) and Titanium (b). Measured TCR of VO $\mathrm{X}_{X}$ and Ti is $-1.16 \% / K$ and $0.21 \% / K$, respectively. Any potential contribution of the contact points has not been isolated from the material TCR and therefore this graph is the sum of both.

\section{CONCLUSIONS}

The detectivities of adaptive microbolometers have been measured vs. frequency in high and low sensitivity states. These bolometers have been designed to have extended dynamic range to handle both very hot and cool areas within the same image. The high sensitivity state corresponded to normal microbolometer operation. In the low sensitivity state, a portion of the support beam was actuated to be partially in contact with the substrate and thereby increased the thermal conductivity and reduced the detectivity. We reported that the thermal conductance of a typical device could be changed by 1.25 orders of magnitude, the responsivity by one order of magnitude, and the detectivity by 1.2 orders of magnitude.

\section{ACKNOWLEDGEMENT}

This work was supported by Defense Advanced Research Project Agency under Grant No. F33615-00-1-1625. The authors would like to thank the staffs at Nanofabrication center at the University of Minnesota.

\section{REFERENCES}

1. R. A. Wood, "Monolithic silicon microbolometer arrays", Ch. 3 in Uncooled Infrared Imaging Systems and Arrays, Ed. P. W. Kruse, and D. D. Skatrud, Semiconductors and Semimetals, vol. 47, Academic Press, New York, 1997, pp. 43-121.

2. J. A. Cutts and R. W. Capps, "Space science applications of infrared detector technology: a review", Proceedings of $4^{\text {th }}$ International Conference of Advanced Infrared Detectors and Systems, 6/5-7/90, (1990), pp. 125-131.

3. P. W. Kruse, L. D. McGlauchlin, and R. B. McQuistan, "Elements of Infrared Technology", Ch. 9, Wiley, New York, 1962.

4. D. E. Burgess, P. A. Manning, and R. Watton, "The theoretical and experimental performance of a pyroelectric array imager", Proceedings of SPIE, 572, 2 (1985).

5. I. H. Choi and K. D. Wise, "A silicon-thermopile-based infrared sensing array for use in automated manufacturing", IEEE Transanction Electron Devices, ED-33, 72 (1986)

6. R. A. Wood, C. J. Han, and P. W. Kruse, "Integrated uncooled infrared detector imaging array", Technical Digest of the 1992 Solid-State Sensor and Actuator Workshop, Hilton Head Isl., SC, 6/2-6/92, Transducer Research Foundation, Cleveland (1992), pp. 132-135.

7. P. C. Shan, Z. Celik-Butler, D. P. Butler, and A. Jahanzeb, "Semiconduting $\mathrm{YBaCuO}$ thin films for uncooled infrared bolometers", Journal of Applied Physics, 78, 7334 (1995).

8. M. Almasri, D. P. Butler, and Z. Celik-Butler, "Self-supporting uncooled infrared microbolometers with low-thermal mass", Journal of Microelectromechanical systems, 10, 469 (2001).

10. R. N. Supino and J. J. Talghader, "Electrostatic control of microstructure thermal conductivity”, Applied Physics Letters, 78, 1778 (2001).

11. W.-B. Song and J. J. Talghader, "Adjustable responsivity for thermal infrared detectors", Applied Physics Letters, 81, 550 (2002).

12. W.-B. Song and J. J. Talghader, "Interface thermal conductance for microactuated devices", Technical Digest of the 2002 Solid-State Sensor and Actuator Workshop, Hilton Head Isl., SC, 6/2-6/02, Transducer Research Foundation, Cleveland (2002), pp. 329-332.

13. W.-B. Song and J. J. Talghader, "Thermal contact conductance of actuated interfaces", Applied Physics Letters, 81, 1216 (2002).

14. P. Erickson, J. Y. Andersson, and G. Stemme, "Thermal characterization of surface-micromachined silicon nitride membranes for thermal infrared detectors", Journal of Microelectromechanical systems, 6, 55 (1997). 